\title{
LA PRIORIDAD DE LA SUSTANCIA EN LA PRIMERA METAFÍSICA DE ARISTÓTELES*†
}

\author{
FABIÁN G. MiÉ \\ CONICET \\ Universidad Nacional de Córdoba \\ fgmie@ffyh.unc.edu.ar
}

RESUMEN: Parte de las diferencias entre los dos modelos ontológicos de Aristóteles, o sea, el de Categorías y el de Metafísica VII-IX, coincide con el desarrollo de una fundamentación de ciertos conceptos operativos. Uno de ellos es el de 'prioridad' en la noción de sustancia primera. Tras formalizar laxamente las distintas significaciones de 'prioridad' intento demostrar que esa ausencia de fundamentación está relacionada con los límites del primer esencialismo aristotélico y con un problema que domina la relación objeto-atributo. Explico este problema que hay en la fundamentación de la 'inherencia' como dependiente de la pervivencia en la primera metafísica del modelo platónico para la relación entre universales e individuos, es decir, como un problema en la justificación de los términos sortales en función identificadora. Sugiero finalmente que la transición al segundo modelo ontológico se realiza cuando se aclaran las condiciones de posibilidad de la identificación de objetos.

PALABRAS CLAVE: Aristóteles, metafísica, sustancia, prioridad

SUMMARY: Some differences between Aristotle's two metaphysical models (the one in Categories and the one in Metaphysics VII-IX) concurs with the

* In memoriam profesora Luly Horenstein.

${ }^{\dagger}$ Este escrito es parte de una investigación llevada adelante en el marco de una beca posdoctoral del Consejo Nacional de Investigaciones Científicas y Técnicas (Argentina) sobre la primera metafísica de Aristóteles. El mismo se halla estrechamente relacionado con otro anterior acerca del modelo esencialista en la distinción objeto-atributo dentro de la primera teoría de la sustancia, pero puede tomarse por separado. Cfr. "La paradoja de la esencia en la primera metafísica de Aristóteles", Diálogos 82 (en prensa). Quiero agradecer a mi director, el Prof. Dr. Osvaldo Guariglia, por su constante apoyo y guía. Al Prof. Dr. Alejandro Vigo quisiera expresarle aquí también mi gratitud por su gentileza al enviarme sus dos textos publicados sobre prioridad y por el intercambio epistolar. El evaluador de este artículo me hizo útiles sugerencias e indicaciones puntuales sobre algunos argumentos y varias formulaciones de este texto. Quisiera reconocer también el interés de los editores al haberme hecho llegar el reciente artículo de Makin [2003] que discute la prioridad de la sustancia en la metafísica madura de Aristóteles. 
development of an appropriate foundation of some operative concepts. One of them is the concept of 'priority' within the notion of primary substance. After a vague formalization of the different meanings of 'priority' I try to demonstrate that this lack of foundation is related to the limits of Aristotle's first essentialism as well as to a problem ruling the object-attribute-relationship. I explain the problem concerning the foundation of the 'inherence' as depending on the survival of Plato's explanatory model of the universal-particularrelationship, that is as a problem arising from the justification of sortal terms in their identificatory function. Finally I propose that the transition from the first ontological model to the second one is accomplished when Aristotle clarifies the conditions of the possibility to identify objects.

KEY WORDS: Aristotle, metaphysics substance, priority

\section{Prioridades y funciones de sortales en la teoría de los compuestos accidentales}

La teoría de los compuestos accidentales aristotélica comienza genuinamente cuando se vislumbra que las entidades a que refieren enunciados estándar — como los que intenta aclarar la doctrina de las categorías a través de la distinción de dos tipos de predicación, tres clases de términos que entran en ellos y cuatro tipos de entidades que ellos suponen (cfr. Cat. 1; 2)están compuestas de ciertas 'partes', cuya relación con el sujeto de que se predican es de un tipo distinto del que caracteriza a otras propiedades que también se predican del mismo sujeto. Asociada a esta distinción de propiedades se halla otra fundamental, pues Aristóteles reconoce que las propiedades dependen de un objeto y se refieren a él y son una clase de entidades no equiparable a los objetos. Un aspecto característico de las partes mencionadas consiste en que la propiedad que ellas representan depende no sólo en su existencia - como cabe a las segundas propiedades antes mencionadas, es decir, las sustancias segundas o universales sustanciales-, sino también en su definición de esa otra clase de entidades identificadas con el sujeto en enunciados de tipo estándar. Esto ya está presente en el pasaje de Tópicos I 9 que introduce las categorías en vinculación con los cuatro predicables, aunque ciertamente no obtiene allí un desarrollo teórico suficiente. Ese paso próximo en la teoría está reservado a las Categorías. Pero tal reformulación da como resultado que lo existencialmente anterior sea también lógica- 
mente anterior ${ }^{1}$ —esto último vale en cuanto su noción está incluida en la definición de las propiedades que se denominan justificadamente 'accidentes' al ser consideradas precisamente como accidentes-del objeto.

La noción del objeto está incluida en la definición de las propiedades denominadas 'accidentales' en la medida en que esta última clase de entidades no agrupa propiedades que se presentan desarticuladas o meramente acopladas y añadidas al objeto, ni definen propiedades que, aun sin poder existir separadas, semánticamente pueden separarse o disociarse de aquello a lo que aparecen atribuidas en ciertos enunciados. Por el contrario, se menciona el objeto cuando se formula una descripción o determinación conceptualmente completa de las mismas, es decir, no una determinación restringida a especificar su identidad semántica — en la respuesta a la pregunta por el 'que es' cuando esas entidades son tomadas por sus nombres abstractos (por ejemplo, 'blancura', 'justicia', en lugar de los parónimos correspondientes 'blanco' y 'justo')—, suponiendo erróneamente que esa identidad podría estar disociada de la función ontológica de estas entidades. Es precisamente atendiendo a esa función de las entidades accidentales - expresada en predicados que remiten a un objeto y que, en tal sentido, son incompletos, algo que se hace visible en su forma parónima - como estas 'partes' deben definirse en su referencia precisa al objeto con el que guardan una relación conceptualmente determinable como inherencia (cfr. Cat. 2, la 24-25). Este concepto debe ser precisado en su distinción de la mera dependencia que cabe a propiedades cuya definición no incluye la de los sustratos individuales, sin los cuales empero, ellas mismas tampoco pueden existir (los universales sustanciales). ${ }^{2}$

${ }^{1}$ Para las nociones de anterioridad $c f r$. aquí infra 2.

${ }^{2}$ Establecer una distinción conceptualmente precisa entre 'dependencia' e 'inherencia' no es una tarea que pueda abordar en este artículo; pero por el lado de la clarificación de las prioridades que operan en la teoría aristotélica de los compuestos accidentales puede echarse algo de luz también sobre las distintas relaciones asimétricas que guardan con el objeto las dos clases diversas de entidades (sustancias universales y entidades no sustanciales) que están involucradas en esas diferentes relaciones. 
Las 'partes' accidentales ${ }^{3}$ son reconocidas por Aristóteles cuando descubre su doble posterioridad: existencial y lógica. Algo lógicamente posterior no puede ser explicado en su acontecimiento a partir del contenido conceptual de aquello de lo que depende, en la medida en que eso de que depende no lo incluye en su propia determinación. Así, un evento es accidental cuando su causa no puede hallarse en la determinación de lo que lo produce ni es explicable por recurso a la intencionalidad del agente. La 'causa' de un accidente no lo explica como un evento necesario, derivado de las determinaciones relevantes de su actor o portador. En tal sentido, el accidente carece de causa, es fortuito en cuanto su productor no extrae ese evento de las determinaciones propias (o de sus deliberaciones, en el caso de la acción); dicho evento no acontece por el objeto (o sujeto) del que depende, sino por otro, con lo cual el propio concep-

${ }^{3}$ En este contexto escribo constantemente la palabra 'partes' entrecomillada debido a que la descripción de esta clase de entidades en Cat. la 24 (mè hos méros) las distingue expresamente de las partes: “(...) en un sujeto digo que es lo que (i) cabe en algo no como (una) parte y (ii) es imposible que sea separado de aquello en lo que es" (la 24-25). La caracterización de las entidades que son-en un sujeto como diferentes de lo que son 'partes' de una sustancia tiene allí un valor doctrinario ya que las entidades inherentes, para ser accidentales, no deben ser explicadas 'como partes', entendiendo en este último uso 'partes físicas'. Éstas no son accidentales, sino, de algún modo, componentes sustanciales. Pero quiero, de una manera un tanto provocativa, hablar de 'partes accidentales' en el marco de referencia de la teoría de los compuestos accidentales a fin de contraponer un poco más agudamente el análisis aristotélico de los componentes de una sustancia - siempre en el contexto de la primera metafísica - con el análisis elementarizante llevado a cabo por Platón, que recurre al establecimiento de entidades anteriores en cuanto son partes de un todo. Mi tesis al respecto, que sólo parcialmente intento justificar en este trabajo, es que la teoría de las categorías aristotélica surge motivada por un rechazo del análisis platónico de las partes sustanciales y constituye una ontología alternativa a la platónica que aspira a reducir los compuestos - entendidos como entidades posteriores - a entidades más simples y, en tal sentido, anteriores. Pero allí no vale entre las entidades anteriores y posteriores lo que Aristóteles entiende como una distinción de tipos de entidades. Para la discusión de Cat. 2, la 24 cfr. Oehler [1997], pp. 225 ss. Sobre el método elementarizante platónico y su crítica por parte de Aristóteles $c f r$. Cattanei [1990]. 
to del accidente no se formula apelando a una determinación esencial y necesaria.

El tipo de términos que se usa para denominar objetos, es decir, entidades aclaradas por Aristóteles como independientes y anteriores, se clasifica como un designador de objetos; éstos no dependen de otra cosa en la determinación de su propia identidad. El análisis de la sustancia no lleva a Aristóteles al descubrimiento de partes o elementos más sustanciales, en la medida en que ellos serían más independientes en comparación con otras entidades — en Platón, las ideas - inicialmente determinadas como sustancias. Por el contrario, más sustanciales son, en el marco del primer modelo ontológico de Aristóteles, las entidades que no se atribuyen a nada distinto de sí mismas; es decir, no son parte de otra cosa o no incluyen necesariamente otra cosa en su propia determinación. La prioridad de la sustancia individual, dentro del modelo ontológico de Categorías, está asociada al reconocimiento de criterios de su contraposición con las entidades excluidas del ámbito sustancial. En efecto, accidentes individuales y universales se distinguen de las sustancias primeras por el hecho de que los términos que designan a los dos primeros no son usados como denominadores de objetos. Los objetos se caracterizan por no necesitar otra cosa para existir. Aquellas entidades, entonces, se predican de objetos que no necesitan otra cosa para ser identificados; por consiguiente, estos objetos tampoco se dicen de otra cosa en el sentido en que se dicen de algo distinto los designadores de accidentes. ${ }^{4}$ La noción de sustancia primera delimita una clase de entidades que ya se halla completa en su identidad cuando se la determina mediante cualquier término accidental; en efecto, mediante la información que se da a través de un término accidental no se está en mejores condiciones de identificar un objeto. Esa clase de términos desempeña una función lógica claramente atributiva y contribuye a una descripción del objeto que se efectúa suponiendo la plena determinación de su identidad.

${ }^{4}$ En cierto sentido, también los nombres de las sustancias segundas se dicen de otra cosa al predicarse de instancias que son distintas de esos universales. 
Aristóteles se sirve de un análisis de la atribución para distinguir criteriológicamente el tipo de propiedades inherentes (Cat. 2, la 24-25), pues inhieren en el sujeto las propiedades que - descritas lingüísticamente - no se atribuyen en su identidad al sujeto ( $c f r$. 5, 2a 27-34). De ellas se distingue la segunda clase de propiedades que considera Cat. (cfr. 2a 19-27), por el hecho de que estas últimas propiedades contribuyen a la identificación del sujeto de que se dicen. Apelando a este criterio de la contribución a la identificación del sujeto se puede establecer una diferenciación en el ámbito de esas propiedades ( $c f r .2 \mathrm{~b}$ 7-17), pues entre ellas hay algunas que contribuyen más que otras a la identificación del sujeto (proximidad especificativa) en cuanto la identificación de algo se hace aquí apelando a su pertenencia a una clase natural o especie. Para todas esas propiedades vale por igual, sin embargo, que su definición se dice del sujeto. Frente a otras propiedades, que pueden describirse lingüísticamente como sortales en función identificadora, existe una segunda clase, cuya no contribución a la identidad del sujeto se prueba en que la definición de esos predicados no se dice nunca del sujeto. Estrictamente, tenemos, en este caso, una clase de atribución del nombre que debería distinguirse de la predicación. Ciertamente, conforme al modelo de la predicación universal, la atribución al sujeto del nombre del accidente ('Sócrates es blanco') no puede explicarse como una 'predicación' stricto sensu; puesto que, en este caso, el contenido conceptual del predicado gramatical no determina al sujeto tal como cuando del sujeto se dice la definición del predicado. En el caso de enunciados como 'Sócrates es justo' o 'Sócrates es blanco', Aristóteles admite que sólo el nombre de la propiedad cualifica al sujeto, en cuanto la identidad del mismo no se ve modificada ni recibe aporte alguno en su contenido conceptual a través de esa atribución. Es entonces la validez, conservada por Aristóteles, del modelo platónico de la predicación universal lo que empuja hacia la zona de la mera cualificación a la predicación de un accidente; e igualmente es la misma teoría de la predicación la que obliga a trazar una firme distinción entre identificación y (mera) atribución. Pero, además, es la 
falta de una clara conciencia de esta distinción y la ausencia de una teoría de la predicación desarrollada en esa dirección lo que empuja a las sustancias segundas a la zona de la mera atribución, de lo cual es testimonio la dificultad que tienen las Categorías para precisar la relación entre sustancias segundas y primeras, distinguiéndola de la relación entre estas últimas y los accidentes.

Cualquier usuario calificado del lenguaje ordinario está en condiciones de indicar a qué tipo de objetos apuntar cuando tenemos un término sortal (por ejemplo, 'hombre') en posición predicativa, mas aplicado a la identificación de un individuo ('Sócrates es hombre'). Es parte de nuestra competencia en el uso de sortales la capacidad de indicar el tipo de individuo que es miembro de la clase — clase que, en otro uso, el mismo sortal denomina, por ejemplo, en 'todos los hombres son mortales'-, así como de distinguir mediante ciertas características (más o menos notorias y más o menos claras y numerosas) los individuos de una especie respecto de los de otra relacionada genéricamente con ella.

Pero con esto Aristóteles ha tomado a los individuos en las Categorías como entidades que asumen una identidad y a sus denominadores como términos que, nombrando al individuo, lo toman ya qua específicamente determinado. Con esa operación marcha de manera teóricamente apresurada la primera metafísica, pasando por alto la explicación de la diferencia que existe entre un designador de individuos, por ejemplo, 'Sócrates', y otro de similar función pero con distinto contenido significativo, por ejemplo, 'este hombre'. Con otras palabras, el primer modelo ontológico de Aristóteles no elabora una teoría paralela a la de los compuestos accidentales, que tendría que explicar - de manera similar a como la de los compuestos accidentales justifica la prioridad del sujeto en predicaciones accidentales estándar orientándose al uso atributivo de sortales - la prioridad del sustrato en cuanto algo determinado, y con ello que la identidad de esa clase de entidades no puede determinarse recurriendo a la definición de su esencia en cuanto ésta sea tomada como algo separado de la cosa definida. Esta diferencia hace que 
las Categorías den por supuesto en el concepto del individuo su identificación, sin empero justificar su aprehensión específica del mismo, o sea, el hecho de que Sócrates es sustancia primera no porque sea ñato o sea justo o sea especialmente atractivo para los jóvenes que visitan los gimnasios, sino porque no es otra cosa que hombre, es decir, un individuo de un tipo determinado, un tóde ti. Lo que está ausente de la primera teoría aristotélica de la sustancia es una justificación de la identidad completa del individuo, siendo que parte de su carácter sustancial lo debe a su determinación específica. En correspondencia, falta también allí una justificación de la sustancialidad de los universales sustanciales, en cuanto la asunción del papel identificador por parte del individuo obliga a que sortales como 'hombre' y 'animal' se prediquen de entidades ya completamente identificadas, y, en tal sentido, la contribución de los universales sustanciales a la identificación del sujeto - lo que caracteriza la función del término predicativo que entra en la predicación universal - resulta superflua.

Aristóteles no alcanza a distinguir claramente la predicación que es preciso reconocerle a sortales como los mencionados - siempre que ellos quieran ser sustancias-, predicación que debe ser diferente de la que tenemos en la atribución de un universal no sustancial ('cultura', 'blancura') al mismo sujeto. Pues la primera metafísica no justifica la diferencia existente entre ambos tipos de predicados ni distingue su diferente uso; y ello porque unos y otros se aplican a entidades ya completamente individualizadas. La 'crisis de sustancialidad' que esto conlleva para los universales de la primera categoría implica que la posterioridad de las sustancias segundas las aproxima a la característica de las entidades no sustanciales. Además, esta posición de las propiedades universales sustanciales y de sus denominadores, que degrada, por lo antedicho, a esas propiedades a la función lógica de la atribución, está en contradicción con la predicación universal, dentro de la cual aquéllas resultan lingüísticamente articuladas. Por esa predicación, Aristóteles califica las propiedades universales como sustancias segundas en virtud de que determinan la identidad del objeto (cfr. Cat. 5, 
2a 14-19: "Entidades secundarias se dicen aquellas especies a las cuales pertenecen las entidades que se dicen primariamente, tanto esas especies, como los géneros de esas especies. Por ejemplo, el hombre particular pertenece a la especie hombre, y el género de esa especie es animal. Secundarias entonces se denominan estas entidades como (el) hombre y (el) animal").

¿Ha pagado Aristóteles un precio demasiado alto en la defensa de su posición antiplatónica al erigir sin concesiones a los individuos como única clase de sustancias y al relegar indiferenciadamente a toda otra clase de entidades? Antes bien que debido a un antiplatonismo parece correcto explicar la falta de una distinción suficiente entre sortales en función identificadora (' $x$ es un hombre') y otros en función meramente atributiva (' $x$ es blanco') como una posición que depende de un resabio platónico presente en la teoría aristotélica de la predicación y del universal que restringe la fundamentación de la prioridad perteneciente a los individuos. Dicho brevemente, tal resabio resulta perceptible en esa posición según la cual los universales, en general, no contribuyen a la identidad de los particulares a riesgo de equipararse ontológicamente con ellos por el hecho de que, si determinasen la identidad de $\{x, y, z\}$, entonces no podrían distinguirse de los individuos en cuanto no tendrían una extensión mayor que ellos. En efecto, la predicación universal platónica que atribuye $F$ a $x$ está obligada a degradar al particular para mantener la separación: $x$ es sólo en cierto grado $F$ para que $x \neq F$. Si no se cumpliera esta condición gradual para todos los particulares sensibles, entonces $x$ no sería distinto de $F$ o $F$ y $x$ serían equiparables. La separación y la gradación - a la cual corresponde la incorrecta aprehensión de las formas como particulares perfectos- buscan garantizar la universalidad de las formas; es decir, el hecho de que ellas abarcan una multiplicidad de particulares constituyendo su aspecto común. Ahora bien, la separación determina que sólo tiene estrictamente identidad la forma $F$, mientras que el particular sensible posee una identidad degradada. Esto significa no sólo que $x$ es menos $F$ que la $F$-idad. La consecuencia que para el platonismo se extrae a partir de la separación de las formas en lo que concierne a la 
identidad indica que $x$ no es nunca completamente idéntico ni por tanto resulta estrictamente identificable mediante una definición, ya que carece de la determinación y la permanencia o estabilidad que son condiciones necesarias para la identificación de un objeto.

Lo que aquí me interesa destacar partiendo de esta propuesta de interpretación de la teoría platónica de las formas, tal como ésta es vista desde una perspectiva aristotélica, es que la ontología de las dos clases de sustancias, presente en Categorías, conserva en la prioridad inconclusa de los individuos un aspecto de la separación platónica de las sustancias cuando Aristóteles toma los particulares sustanciales como entidades a cuya determinación no prestan la contribución adecuada los universales sustanciales. La razón de esto se halla en que los particulares sustanciales ya son plenamente determinados. Una consecuencia de esta posición es que los universales de la primera categoría son sustancias: deúterai ousíai. Es decir, una superposición de sustancias en la primera categoría expresa que los universales y los particulares son entidades completamente determinadas, y da cuenta a la vez de la falta de una fundamentación de la pertenencia de un individuo - si lo consideramos en sus características particulares, que es precisamente aquello que no puede justificar el primer modelo ontológico de Aristótelesa una determinada especie. El problema es serio para Aristóteles si se tiene en cuenta que él rechaza tempranamente y sin concesiones la participación como una explicación adecuada de esa relación. ${ }^{5}$

Este aspecto de la predicación universal platónica que pervive como resabio en la aristotélica puede ser caracterizado adecuadamente en el marco del hèn epì pollôn. ${ }^{6}$ En el platonismo, el peso ontológico cae sobre los universales. A consecuencia de ello, la distinción entre las diferentes funciones antes mencionadas que desempeñan los términos sortales no aparece como

${ }^{5}$ La anterior interpretación de la teoría de las formas platónica y particularmente de la predicación universal es controvertible, pero no puedo aquí tratar de explicarla.

${ }^{6}$ Para la crítica aristotélica al argumento del uno sobre los múltiples $c f r$. Leszl [1975], VI y II; Fine [1998] 8. 
una tarea relevante. Pero esto es, según Aristóteles, resultado de una aprehensión incorrecta de los enunciados donde entran los sortales. La metodología de la definición representada por la diaíresis es un ejemplo de ello: Platón no dispone allí de ninguna herramienta metodológica para distinguir claramente (i) universales bajo los que se incluyen especies inferiores (definienda) respecto de otros (ii) universales cuyo definiendum no se incluye en la determinación del objeto, más allá de que estos últimos universales guarden efectivamente una cierta relación de atribución con el objeto. El método platónico plantea únicamente el problema de manera marginal cuando afronta la dificultad de distinguir entre genuinas especies (êेdos) y meras partes (méros), donde se hace necesario disponer de ciertos criterios - provistos por la doctrina de las partes de la alteridad - para diferenciar un conjunto de rasgos carentes de genuina razón de unidad con respecto a otro conjunto de características-parte constituyentes de un eîdos-total, unificado éste por una articulación e irreducible él mismo a otras propiedades específicas. De cualquier manera, este serio problema reconocido por Platón no da lugar al planteamiento de la distinción criteriológica de propiedades universales esenciales y no esenciales, que está en el origen del esencialismo aristotélico. ${ }^{7}$

De acuerdo con la teoría de la predicación universal expresada en el 'uno sobre muchos', un universal se distingue de un individuo en virtud de que se dice de muchos particulares. Ahora bien, Platón llegó a establecer que la condición de la extensión era sostenible sólo si el universal se distingue ontológicamente de los particulares de que se enuncia en cuanto él mismo constituía un cierto objeto. Platón no disoció la predicación universal

${ }^{7}$ Para la doctrina de las partes de la alteridad y la teoría platónica de la negación cfr. Lee [1972]; Owen [1971]; Szaif [1998³], pp. 412-509; O'Brien [1995], pp. 43-102, presenta una discusión que mantiene algunas posiciones discordantes respecto de la de Owen; para la fundamentación de la distinción entre especies y partes en la doctrina de las partes de la alteridad $c f r$. brevemente Mié [2001], IV; para la distinción ê̂dos/méros cfr. Marcos [1995]; Moravcsik [1973a] y Cohen [1973] destacaron el papel central que esta distinción tiene para el exitoso funcionamiento del método, así como las falencias del mismo en tal sentido. 
de la condición de singularidad ontológica, y así, para seguir manteniendo al universal en la predicación correspondiente, tuvo que separarlo ontológicamente de los particulares sensibles, erigiéndolo como un particular con un rango superior derivado éste del modo en que se predica, es decir, del hecho de que es el primer denotado del nombre de la propiedad que se aplica a las cosas particulares. Con ello, Platón hace del universal un 'particular perfecto'. ${ }^{8}$ El universal separado, de esta manera, era preservado de los avatares de la vida sensible; pero, a consecuencia de ese destino, no podía contribuir a la identificación de las cosas sensibles. Platón llegó a una solución de compromiso, cuando entendió la predicación universal como un garante de la ciencia, pero, no pudiendo admitir que entidades no universales fueran científicamente accesibles, debió restringir la influencia de las ideas — de las que participan los particulares sensibles, a consecuencia de lo cual forman parte de una misma clase como miembros de una especie única - sobre los individuos a un aporte que, en términos de teoría de la predicación, podría caracterizarse como una 'predicación accidental irrestricta'.

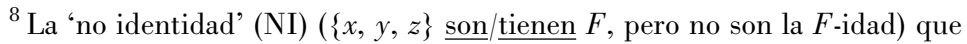
asume esta teoría implica que la propiedad sensible de las cosas particulares $(f)$ no cabe a sus portadores en virtud de ellos mismos, sino en virtud de una forma $(F)$ numéricamente diferente de los portadores y de su propiedad sensible. $F$ existe, por ello, necesariamente separada (asunción de la separación $=$ S). Sobre la NI $c f r$. Fine [1998], pp. 15-16. La unidad numérica (UN) de las ideas es un teorema que se deriva de la exigencia epistémica contenida en el modo específico en que Platón entiende el 'qué es'. Esta interrogación no se satisface con referentes que son singulares desde un punto de vista meramente específico (UE). Las ideas deben ser numéricamente singulares o simples para evitar la diferencia o alteridad, de la cual la 'compresencia' representa una versión radicalizada en el caso de la compresencia de propiedades contrarias. El rasgo de la UN de las ideas permite inferir la $\mathrm{S}$, pues de lo contrario las ideas deberían existir mezcladas como una parte física o material de las cosas de que se predican metafísicamente. Fine, pp. 60 y s. (con nn.), llama oportunamente la atención sobre ciertas premisas faltantes en algunas habituales reconstrucciones de la $\mathrm{S}$ de las ideas.

${ }^{9}$ Por lo dicho anteriormente resulta evidente que la accidentalidad es contraria a los requisitos de la clase de predicación universal que Platón define para las ideas; y, sin embargo, es la única opción factible obligada por la separación. 
Uno de los principales resultados de la inversión del platonismo que conlleva la anterioridad de los individuos en el primer modelo ontológico de Aristóteles está dado por la teoría de los compuestos accidentales. Se puede afirmar que las 'partes' accidentales son sólo en cuanto son inherentes al sujeto. Y son inherentes al sujeto al articularse con él, siendo pasibles, con ello, de una modificación de la que el lenguaje da cuenta a través de la paronimia o inflexión en el nombre. Los parónimos expresan un ítem ontológico - caracterizado, ciertamente, a través del lenguaje, pero no un mero 'ítem lingüístico'-10 propio de esta teoría: los compuestos accidentales, es decir, entidades compuestas de un objeto y una propiedad accidental inherente a él. Nuevamente a las Categorías reserva Aristóteles la explicación y fundamentación de esta teoría. La misma permanece dentro de los márgenes de la primera metafísica y define, en ese contexto, la ontología aristotélica derivada de la doctrina de las categorías. Mi objetivo aquí es mostrar que la teoría de los compuestos accidentales reformula las dos prioridades (lógica y ontológica) haciendo de lo primero ontológico, sin inconsistencia, algo lógicamente anterior.

${ }^{10}$ Esta última es una versión a la que dio lugar una observación de Owen [1960], p. 175 — ya desde los primeros comentadores griegos fue discutido si acaso la clasificación de los términos en Cat. 1 alcanzaba meramente a expresiones lingüísticas o, en definitiva, concernía a items extralingüísticos o entidades reales -; pero ya Ackrill [1963], p. 73, y, sobre todo, Oehler [1997], pp. 192 y ss., 101 y s., aclararon que el interés de Aristóteles en Cat. 1 no se dirige a un análisis de los términos disociado de los items extralingüísticos, sino que, más bien al contrario, a Aristóteles no le interesa hacer un análisis y una clasificación de los términos y usos lingüísticos por sí mismos, pues su objetivo - tanto aquí, como en el resto de sus obras del Órganon - está puesto en una clarificación de las entidades a través del medio de su aprehensión, esto es, el lenguaje. Wedin [2000], p. 14, observa acertadamente "Del hecho de que la definición de parónimos sea meramente gramatical no se sigue que los parónimos mismos sean entidades gramaticales." [From the fact that the definition of paronyms is merely grammatical it does not follow that paronyms themselves are grammatical entities.] La inflexión (diaphéronta têi ptósei), que caracteriza y distingue a los parónimos, es un registro lingüístico de una relación asimétrica entre cosas, donde la definición de una se deriva de la definición de la otra. 
Paso ahora a formalizar laxamente los distintos conceptos de prioridad y a tratar de aclarar sus significados para posteriormente considerarlos en función de la anterioridad que le cabe a la sustancia individual en el primer modelo ontológico de Aristóteles. ${ }^{11}$

${ }^{11}$ Antes de ello quiero hacer una observación aclaratoria sobre el discutido tema de la coincidencia entre las dos nociones diferentes de prioridad - es decir, lógica y ontológica - en la clase de entidad que constituye la sustancia primera, y sobre la falta de coincidencia de ambas nociones en el caso de otro tipo de entidades. Desde hace tiempo, los comentadores modernos de Aristóteles han discutido pasajes donde se oponen la prioridad lógica (lógoi) y la ontológica (ousíai). Clásico, en este aspecto, es el texto de Metaph. XIII 2, 1077a 36-b 11, pero cfr. también, por ejemplo, XIII 8, 1084b 2-32. Sin embargo, y contra lo que sugiere una lectura de XIII 2, 1077b 4 (toûto [scil. las dos clases de prioridad, es decir, en la definición y en la sustancia o entidad] dè ouch hámai hypárchei, sobre lo cual advierten Ross [1958], II, p. 415 y Vigo [1990], p. 182 n. 17, traduciendo con la perífrasis "no se aplican coextensivamente", cfr. también Annas [1998], p. 133, y su comentario, pp. 146 y s.) que podría hacer capitular la consistencia entre prioridad lógica y ontológica, Aristóteles no disocia allí ambas prioridades, en general, sino que defiende su coincidencia en el caso de los compuestos accidentales. Para el caso de los objetos matemáticos — lo que se aplica, similarmente, a los objetos de otras ciencias-, en cambio, no vale que la prioridad en la definición y en la entidad o sustancia sean coextensivas - nótese que no hablo, en este caso, de prioridad meramente existencial (para estas distinciones $c$ fr. infra 2.). Y no vale porque la definición de un objeto matemático no determina una entidad separada, o sea, no es la fórmula de la esencia de una entidad que no supone algo distinto de ella. Las definiciones de los objetos matemáticos pueden formularse únicamente bajo la suposición de su separación, es decir, cuando se los toma como si fueran separados. A esto llama Aristóteles 'separación en el pensamiento' (têi noései Ph. II 2, 193b 34 s., 194a 9-12). El producto de esta separación mental coincide con la operación de los matemáticos, quienes, de esa manera, pueden considerar su objeto propio como tal, como si fuera en sí, es decir, separado. Sobre esto cfr. Metaph. XIII 3, 1077b 34-1078a 9, y el tratamiento de Cleary [1995], p. 319 ss. (cfr. en general. su clarificadora discusión, pp. 307-339); sobre la aphaíresis aristotélica $c f r$. en especial Happ [1971], pp. 584 y ss., 587, 591, 600; Annas [1988], pp. 137 s., y 144. Es perfectamente admisible, y no induce al error, que se 'ponga aparte' (ektíthesthai) esa propiedad. La operación mental de abstracción (separación en el pensamiento) considera como separadas propiedades que son inherentes en una sustancia, es decir, en una entidad actualmente separada. Sólo en la medida en que el matemático y los restantes científicos separan conceptualmente el objeto - entre ellos, las propiedades cuantitativas que son objeto de la matemática- de sus ciencias respectivas, 


\section{2. 'Prioridad según la no reversibilidad en la implicación} de existencia', "prioridad según el conocimiento del 'qué es", y 'prioridad según la naturaleza y entidad' en la explicación de la prioridad de la sustancia primera

Considerando las dos principales clasificaciones de la noción de prioridad que tenemos en el corpus aristotélico, esto es, las que se hallan en Cat. 12 y Metaph. V 11, se puede obtener una mejor comprensión del tipo de prioridad que es necesaria para

es decir, en la medida en que actualizan mentalmente sus objetos (como Ross [1958] acentuó, $c f r$. p. 418), proceden a dar de ellos definiciones, tal como éstas se hallan en la operación regular del matemático. En tal sentido, esos objetos llegan a ser lógicamente primeros. Pero son también, y en no menor medida, ontológicamente posteriores, en cuanto no existen como sustancias separadas en acto, sino que en su existencia presuponen un sustrato determinado, en el que inhieren como propiedades accidentales cuantitativas. Un análisis ulterior puede abordar la relación de prioridad-posterioridad, que cabe adjudicar a los compuestos matemáticos mismos, pero ello me llevaría fuera del marco presente (sobre esto $c f r$. Happ [1971], pp. 609 y ss.). Con esta observación aspiro a hacer un poco más comprensible que la 'prioridad' admisible para los accidentes de un compuesto accidental conforma una posición consistente con la separación mental y con la definibilidad que se puede adjudicar a los objetos de las diversas ciencias. Y éste es el motivo genuino por el cual Aristóteles apela a los compuestos accidentales para aclarar el problema planteado acerca de la prioridad en la definición de los objetos matemáticos respecto de las cosas sensibles, en el texto de Metaph. XIII 2, 1077a 36-b 11, tal como lo confirma el paralelo trazado entre las propiedades abstraídas (cuantitativas) y las adicionadas (accidentes en general), cfr. 1077b 9-11. Sobre el sentido de prósthesis allí (añadido de un accidente a una sustancia) cfr. Ross [1958], p. 415 (remite al uso del verbo con dat. en VII 4, 1029b 33); para la 'definición por adición' (ek prosthéseos lógos) cfr. el típico caso de la definición (en VII 5) de un compuesto por sí (1030b 19-20). Para un comentario focalizado en el tema de las prioridades del pasaje de Metaph. XIII 2 cfr. Vigo [1990], pp. 182 y ss. Vigo llama oportunamente la atención sobre que la clasificación de los significados de 'prioridad' en Metaph. V 11 (1018b 34-35; sobre ello cfr. aquí infra) permite abordar la prioridad del accidente en XIII 2: con el vocabulario de V 11 convendría llamar a esa prioridad 'según el conocimiento' (cfr. Vigo [1990], p. 183 y nn. 19 y s.). Acerca de la consistencia entre las posiciones sobre las prioridades de Metaph. VII 1 y XIII 2, cfr. Vigo [1990], p. 187. 
fundamentar el rango prioritario de la sustancia primera. ${ }^{12}$ Esos dos textos, junto a otros muchos donde Aristóteles hace uso de un concepto bien diferenciado de prioridad, muestran inmediatamente que una visión restringida de esa noción no puede aplicarse con éxito al análisis de los conceptos ontológicos centrales de la metafísica aristotélica, ni permite observar con claridad que la prioridad de la sustancia primera no es un postulado de la metafísica aristotélica, sino un teorema ontológico que debe ser fundamentado.

Hay tres significados de 'prioridad'13 que pueden considerarse especialmente para este fin:

(I) Prioridad existencial: Cat. 12 (2) ${ }^{14}$ (14a 29-35): Prioridad

${ }^{12}$ Vigo [1989], 2 y 3, ha comentado los dos textos mencionados; para algunos detalles de esos textos remito a su comentario y a la bibliografía citada en sus notas.

${ }^{13}$ Vigo recuerda que el término 'próteron' o 'prôtos' — para la equivalencia semántica de estas dos expresiones cfr. Bonitz [1955²], 652b 31- posee múltiples significados, es decir, es un pollachôs legómenon. Vigo [1989], 90 n. 1, anota: Cat. 12, 14a 26-27: tetrachôs; Ph. VIII 7, 260b 18: pleonachôs; GA II 6, 742a 19-20 y Metaph. VII 1, 1028a 29: pollachôs. En otros muchos pasajes, sin declarar expresamente esa multivocidad, se la presupone ( $c f r$. Vigo, 90 n. 2), por ejemplo $P h$. VIII 9, 265a 22; $P A$ II 1, 646a 25-b 10; $R h$. II 19, 1392a 15-22; Metaph. VII 13, 1038a 27-28, etc. (los artículos dedicados a próteros y prôtos en Bonitz son sumamente instructivos).

${ }^{14}$ El número entre paréntesis tiene sentido ordinal y refiere al significado de la serie presentada por Aristóteles. Me parece útil y favorable a la claridad intentar, a continuación, una formalización laxa de los restantes usos distinguidos, los cuales no son empero especialmente relevantes para mi discusión sobre la prioridad de la sustancia en este parágrafo.

Cat. 12 (1) (14a 27-29): Prioridad según el tiempo (katà chrónon):

$X$ es anterior a $Y$ según el tiempo, si

(i) $X$ existe en un periodo de tiempo mayor $\left(t_{1}-t_{n+1}\right)$ que $Y\left(t_{1}-t_{n}\right)$.

Cat. 12 (3) (14a 35-b 3): Prioridad según el orden (katà tina táxin):

$X$ es anterior a $Y$ según el orden, si

(i) $(X, Y)$ son elementos (a) de las ciencias o (b) del lógos (composición gramatical y discursiva);

(ii) $Y$ es un tipo de entidad que se inserta en una cierta relación que determina la organización de una cosa; 
según la no reversibilidad en la implicación de existencia (tò mè antistréphon katà tèn toû eînai akoloúthesin):

$X$ es anterior a $Y$ según la no reversibilidad en la implicación de existencia, si

(i) $Y$ existe de tal manera que implica la existencia de $X$;

(ii) la implicación descrita en (i) es un dato que se obtiene a partir del contenido lógico inmediato del concepto de $Y$ (una implicación directa (akolouthề euthùs 14a 31-32));

(iii) la implicación descrita en (i) no es reversible, es decir, no vale que a partir del contenido conceptual de la proposición que afirma la existencia de $X$ se pueda obtener lógicamente de manera inmediata la existencia de $Y$.

(II) Prioridad lógica: Metaph. V 11 (2) ${ }^{15}$ (1018b 29-37): Prioridad según el conocimiento (tò têi gnósei):

$X$ es absolutamente anterior a $Y$ según el conocimiento, si

(iii) e.g. (a) $X$ es un axioma o postulado e $Y$ una proposición, (b) $X$ es una letra e $Y$ una sílaba o $X$ es un proemio e $Y$ una exposición.

Cat. 12 (4) (14b 3-8): Prioridad según el valor (tò béltion kaì tò timióteron) (significado impropio):

$X$ es anterior a $Y$ según el valor, si

(i) $X$ es por naturaleza mejor que $Y$.

Cat. 12 (5) (14b 10-22): Prioridad según la causa:

$X$ es anterior a $Y$ según la causa, si

(i) $X$ explica la existencia de $Y$, y no viceversa;

(ii) e.g. en casos de biimplicación existencial o simultaneidad contingente es posible establecer una asimetría causal entre los miembros relacionados, demarcando a $X$ como anterior a $Y$ en cuanto $X$ explica la existencia de $Y$, y no viceversa.

${ }^{15}$ Siguiendo la sugerencia de la nota anterior, formalizo aquí los restantes usos de 'anterior' distinguidos en Metaph. V 11 (para algunas cuestiones puntuales del texto $c f r$. Vigo [1989], pp. 91-94 nn.

Metaph. V 11 (1) (1018b 9-29): Prioridad según la cercanía al principio de cada género: 
A) (de acuerdo con el concepto (katà tón lógon) (conocimiento conceptual), (i) $X$ es universal e $Y$ es individual (1018b 27-34); (ii) $X$ es accidente e $Y$ es un todo-compuesto accidental (1018b 34-37);

(B) de acuerdo con la percepción (katà tèn aísthesin) (conocimiento perceptual), $X$ es individual e $Y$ es universal.

(III) Prioridad ontológica: Metaph. V 11 (4) (1019a 2-14):

(A) El principio válido en cada uno de los géneros puede fijarse (al) absolutamente y por naturaleza, (a2) relativamente, (a3) por su ubicación, (a4) por algunos sujetos (1018b 9-12);

(B) $X$ es anterior a $Y$ según la cercanía al principio, si

(bl) de acuerdo con el lugar, $X$ está más cerca que $Y$ de un punto o lugar de referencia fijado por naturaleza o al azar (1018b 12-14);

(b2) de acuerdo con el tiempo, tomando el ahora como principio y lo primero, $X$ está, con referencia al pasado, más alejado del ahora que $Y$; y, con referencia al futuro, $X$ está mas cercano al ahora que $Y$ (1018b 14-19);

(b3) de acuerdo con el movimiento, considerando como principio absoluto aquello que origina el movimiento, $X$ está más cerca de ese principio que $Y$ (1018b 19-21);

(b4) de acuerdo con la potencia, considerando ciertos principios diferentes en cada ámbito de entidades, $X$ puede más que $Y$ (por ejemplo, si se toma la voluntad como principio para el caso de los actos humanos, $X$ es anterior a $Y$ si de $X$ depende que $Y$ sea querido, es decir, $X$ pone en movimiento la acción que lleva a realizar $Y)(1018 b$ 22-26);

(b5) de acuerdo con el orden, tomando principios distintos para el caso de distintas clases de entidades organizadas conforme a una cierta relación o razón, $X$ está más próximo al principio (de esa relación) que $Y$ (1018b 26-29).

Metaph. V 11 (3) (1018b 37-1019a 1): Prioridad de las propiedades de las cosas anteriores:

$X$ es anterior a $Y$ según la propiedad de las cosas anteriores, si

(i) $X$ es una propiedad de una cosa (sustancia), y ésta es anterior a aquella cosa de que $Y$ es una propiedad. 
Prioridad según naturaleza y entidad (katà phýsin kaì ousían) (distinción platónica): ${ }^{16}$

$X$ es anterior a $Y$ según naturaleza y entidad, si

(i) $X$ puede ser $\sin Y$, y no viceversa (1019a 3$)$;

(ii) dado que 'ser' tiene múltiples significados (1019a 4-5), es posible aplicar esta prioridad:

(iia) según el esquema de las categorías, ${ }^{17}$ la ousía qua suje-

${ }^{16}$ Aristóteles adjudica a Platón esta distinción en Metaph. V 11, 1019a 4; sobre las dificultades para hallar una referencia exacta en los textos platónicos para esta doctrina $c f r$. la observación de Vigo [1989], p. 93 n. 8 (con referencia bibliográfica, a la cual habría que añadir otras citas de obras de Gaiser, por ejemplo $\left[1968^{2}\right]$, pp. 48, 80, passim). El esquema referido pertenece, seguramente, al conglomerado de "doctrinas no escritas" platónicas. Contra lo que puede parecer, esta prioridad no es equiparable a lo que Owen [1960] llama "prioridad natural" — caracterizada por Owen así: $A$ es naturalmente primero que $B \equiv A$ puede existir $\sin B$ y no viceversa-, pues el dominio de esta noción de raigambre platónica, es decir, su aplicación a la teoría de los compuestos accidentales —una teoría totalmente ajena a Platón- demarca la transformación a que Aristóteles somete a la misma y ello indica que la prioridad natural no puede explicarse como una noción primitiva (Owen) - contrapuesta a lo que Owen llama "prioridad lógica" - que dominaría la primera metafísica de Aristóteles. La clasificación de Owen debe corregirse atendiendo a los distintos usos de 'prioridad' reconocidos por Aristóteles. Con esa corrección se hace necesario redemarcar las distinciones entre los dos modelos ontológicos aristotélicos. Vigo [1989] y [1990] propuso también una corrección de este tipo.

${ }^{17}$ Aristóteles no recurre, en esta oportunidad, a alguno de los habituales giros con que hace referencia a las categorías; no obstante, es indudable que se trata de eso. Cfr. Ross [1958], I, pp. 317 y s. Esta aplicación de la prioridad se hace al segundo significado de tò ón distinguido en Metaph. V 7, 1017a 22-30. Allí se clasifican las entidades por sí apelando a las distinciones que surgen dentro de esa clase cuando se aplica el esquema de las categorías. O sea, que Metaph. V 7 (2) da todas aquellas entidades por sí que son identificables a partir de los distintos significados obtenidos de las diferentes figuras de la predicación. Dentro del cuádruple significado de 'ser' (ser-por sí, distinto de ser-accidente, ser-verdadero y ser-en potencia y acto) se distinguen diferentes maneras en que puede expresarse lo-que-es-por sí; mediante este recurso se establecen distinciones en el conjunto de las cosas que son por sí. Hay tantas modalidades de ser-por sí, cuantas clases de predicación son distinguibles, según el enunciado programático de 1017a 24-25; éste tiene 
to (hypokeímenon) de la atribución accidental es anterior $<$ a los predicados accidentales $>$ (compuestos categoriales) (1019a 5-6);

(iib) según potencia y acto, en el caso de compuestos de elementos distinguibles según la modalidad ontológica de potencia y acto, algo es potencialmente anterior, en cuanto alcanza la existencia actual si el todo del que forma parte se disuelve, por ejemplo, la semilínea respecto de la línea, la parte respecto del todo, la materia respecto de la ousía qua forma (compuestos modales) (1019a 6-11). ${ }^{18}$

Metaph. V 11 (4), restringiéndome ahora a la primera aplicación del concepto de prioridad allí clasificado (iia), es decir, la prioridad de la sustancia en el esquema de las categorías, sostiene la posición madura de VII l, según la cual hay un

como consecuencia la aplicación del análisis categorial a las entidades por sí. Dificultades innecesarias surgen para la interpretación de este texto cuando se entiende que Metaph. V 7 (2) clasifica 'accidentes por sî', suponiendo que los predicados, a los cuales allí se apela para la clasificación, desempeñan la función de nombres para entidades accidentales. Quienes se enredan en esta aparente dificultad confunden injustificadamente (i) la función de los predicados entendidos como nombres para ciertas entidades con (ii) la función de los predicados entendidos como denominadores de propiedades que cualifican al sujeto. Al esclarecimiento de la segunda función sólo se llega mediante la aplicación a los predicados de un análisis según la prioridad que ahora discuto en (iia). Para una discusión de la relación entre categorías y entidades por sí cfr. Calvo [1991].

${ }^{18}$ Según Ferejohn [1981], p. 293, la prioridad lógica habría reemplazado a la ontológica (o natural) (cfr. Metaph. VII 13, 1038b 33) a fin de resolver una posible inconsistencia en la anterioridad que al género le cabe respecto de la especie — de la cual aquél depende para existir, según la posición antiplatónica de ese capítulo. La prioridad del género cumple allí con lo que Ferejohn llama la "condición de inclusión en el lógos", que constituiría una mejora, provista por $A P o$. I 4, 73a 35-b 4, a la verdad definicional o necesaria, entendida en los términos del par excluyente decir-de/ser-en, de Cat. ( $c f r$. S5 reemplazado por S5' en Ferejohn, pp. 287, 292). Esa precisión de APo. puede efectivamente aportar una clarificación de la anterioridad del género, precisión que no aporta la clasificación de Cat. 12 atinente a distintos significados de anterior. Pero una cuestión que habría que discutir más detalladamente es si la prioridad del predicado en la predicación decir-de es, efectivamente, de tipo ontológico o natural. Según Ferejohn, ésa es la posición que reemplazaría la de Metaph. 
sentido de 'ser' primero y ése ese el significado de la sustancia (ousía) dentro de un análisis categorial del ser (tò ón). Ahora bien, Aristóteles llega a esta posición aplicando a un marco desconocido para Platón un esquema que "usaba"19 Platón (V 11, 1019a 4). Lo que se conserva de ese esquema en la aplicación aristotélica permite afirmar que los accidentes no pueden existir si no existe anteriormente el sustrato, pero no viceversa. La prioridad de la ousía en el compuesto categorial es también una prioridad según la determinación que a esa entidad primera le corresponde respecto de la posterioridad que caracteriza a otras determinaciones, en la medida en que ellas no delimitan el 'qué es' del objeto y son, entonces, determinaciones inesenciales. Esta aplicación específica del esquema platónico de la prioridad le permite a Aristóteles demarcar una entidad que no se halla en el mismo nivel que las posteriores — como sí es el caso, en cambio, en el esquema platónico de la división o en el de la sucesión dimensional y en el de los números ideales, donde ese esquema tenía aplicación originalmente-, ya que la posición aristotélica implica la distinción de una entidad lógica y ontológicamente independiente, respecto de otras cuya existencia y esencia depende de las de esa otra entidad. ${ }^{20}$

La dependencia existencial de los accidentes — según la cual hay ciertas propiedades que dependen de un sustrato para existir en la medida en que no son entidades por sí (autosubsistentes) - está indisolublemente ligada, en el segundo

${ }^{19}$ En Metaph. V 11, 1019a 4 leo el texto de Jaeger (cfr. ad loc.): echrêto (imperfecto, en lugar del aor. echrésato) (contra Ross).

${ }^{20}$ Ross [1958], I, p. 318, comenta al respecto y en referencia al ê̂nai de Metaph. V 11, 1019a 3: "Ya que la sustancia es en un sentido más pleno que las otras categorías, es anterior a ellas" [Since substance is in a fuller sense than the other categories, it is prior to them]. Pero, si bien este comentario es, sin duda, correcto, deja demasiado incierto el fundamento de la prioridad, pues ese fundamento no puede considerarse suficientemente expresado apelando a una diferencia gradual - lo que corre el riesgo de remitirnos a una ontología de carácter platónico. Esa clase de diferencia sería más adecuada para describir una ontología que sólo puede establecer entre las entidades explicadas bajo un mismo concepto (todas ideas-sustancias) una mera diferencia de grado. Pero es justamente este análisis el que viene a corregir el esquema de las categorías. 
modelo ontológico de Aristóteles, a una dependencia determinativa. Pues la prioridad de la sustancia en el marco del esquema de las categorías consiste en que hay una entidad cuya esencia - esto debe servir para aclarar el significado de phýsis y ousía en Metaph. V 11 (4iia) - es independiente de ciertas otras determinaciones, y sólo ello explica que estas últimas sean clasificadas como posteriores a y dependientes de la primaria - inesenciales - , como también que sus nombres denoten entidades que no se presentan nunca separadas ni subsisten por sí mismas.

Este doble sentido de la prioridad de la sustancia en el esquema de las categorías es lo que lleva justificadamente dicho esquema y su prioridad, y también el concepto de Cat. 12 (2), a su aplicación programática en la declaración de Metaph. V 7, 1017a 24-25. En 1017a 22-27, Aristóteles expone la relación entre los diferentes esquemas de la predicación y los diferentes significados de 'ser' considerado por sí:

Ser-por sí se dice precisamente de tantas maneras, cuantas significa <'ser'> en los esquemas de la predicación. Pues de cuantas maneras se predica, de tantas maneras significa 'ser'. Ahora bien, ya que de los predicados unos significan qué es, otros cuál, otros cuánto, otros en relación con algo, otros hacer o padecer, otros dónde, otros cuándo, por consiguiente 'ser' significa lo mismo que cada uno de estos <tipos de predicado>.

El sentido aristotélico de la prioridad de la ousía genera una ontología diferente y da lugar al desarrollo de la prioridad de la ousía qua sujeto categorial en Metaph. VII 1. La prioridad de Cat. 12 (2) no explica por sí sola la prioridad de la sustancia, tal como la formula Metaph. VII 1. La ousía reconocida en VII 1 es primera no sólo ni sobre todo en virtud de que a partir del concepto de ciertas propiedades afirmadas como existentes se deduzca la existencia de una entidad especial. Esto no es incorrecto si se da como una caracterización general de la posición de la sustancia tanto en Categorías, como en Metafísica, pero es insuficiente para dar cuenta de la prioridad sustancial del objeto en la metafísica madura, es decir, resulta insuficiente para 
explicar la prioridad que cabe atribuirle a una entidad en razón de la completud de su determinación o de que ya era lo que es con anterioridad a la atribución de otras propiedades. La ousía de Metaph. VII 1 es primera entonces no meramente porque no se predica de otras entidades, mientras que todas las otras se predican de ella. Este último criterio, que puede aplicarse a la sustancia primera de Cat. $5-\mathrm{y}$ al que se alude también en Metaph. V 8, 1017b 13-14- no alcanza para fundamentar lo que implica la prioridad de la sustancia dentro del esquema de las categorías en su aplicación a la ontología madura de VII 1 , y ni siquiera es suficiente para fundamentar la prioridad de las sustancias individuales en Categorías. Es sólo mediante la explicación referida a que hay ciertas determinaciones que suponen en su propio concepto la determinación y la existencia de otra clase de entidad cuando se alcanza la posición de la ousía en Metaph. VII. ${ }^{21}$

La prioridad de la sustancia en Metaph. V 11 (4iia) no puede meramente explicarse recurriendo a la anterioridad existencial

${ }^{21}$ Ésta es la razón principal por la cual he tratado de establecer una diferencia conceptualmente clara entre lo que he llamado prioridad existencial y prioridad ontológica. No favorece el establecimiento de esta distinción describir Metaph. V 11 (4) en términos de mera "condición de independencia" (CI) (Makin [2003]). Por consiguiente, no parece adecuado plantear el problema característico de la metafísica madura de Aristóteles concerniente a la anterioridad sustancial en el orden del acto, que es suscitado en Metaph. IX 8, aplicando el concepto de Metaph. V 11 (4iib) como si éste fuera idéntico a la prioridad existencial. Hacerlo puede dar lugar a la acusación según la cual Aristóteles no apelaría a la CI — que básicamente coincide con la prioridad existencial - para fundamentar la prioridad del acto que corresponde a la sustancia en Metaph. IX 8, 1050a 4-105la 2. Es probable, en cambio, que el concepto de prioridad al que hay que recurrir para fundamentar la prioridad sustancial del acto incluya los factores mediante los que Aristóteles explica la prioridad del acto: la relación teleológica que vincula potencia y acto. Witt [2000], pp. 217 y s., formula una objeción como la señalada y Makin [2003] discute y valida la CI en su relevancia para IX 8 haciendo un extenso análisis de Metaph. V 11, 1019a 2-14 (= (4iib)). Makin [2003] (cfr. 3.) da una explicación no restringida de ese texto destacando correctamente en él los aspectos teleológicos vinculados a la anterioridad según la generación y la destrucción (1019a 12-14). Esta consideración cae fuera de mi tratamiento limitado a la primera metafísica aristotélica que no incluye el esquema modal de potencia-acto. 
de ciertas entidades obtenidas a partir de la implicación semántica entre dos proposiciones existenciales. Esto es bien destacado por $\mathrm{Vigo}^{22}$ como una diferencia importante entre Cat. 12 (2) y Metaph. V 11 (4iia). En efecto, la anterioridad sustancial es formulada por Aristóteles como una alternativa al esquema semántico de origen platónico, según el cual la implicación semántica entre, por ejemplo, cuerpo-superficie y superficie-línea da lugar a la postulación de la anterioridad existencial de aquello que en el platonismo son los elementos-parte de que se componen las cosas, que son explicadas así como posteriores. Aristóteles rechaza un análisis elementarizante de ese tipo en el que se da con las sustancias aplicando el mencionado criterio de prioridad.

Asimismo, la prioridad de implicación existencial de Cat. 12 (2) no alcanza a dar cuenta de la dependencia que caracteriza a la relación sustancia-accidentes y que instaura una heterogeneidad entre ambas clases de entidades, además de provocar que ellas no puedan ser colocadas en el mismo nivel. Creo que no es correcto interpretar que la genuina y más importante diferencia entre ambos textos reside meramente en que el de Metaph. formula dos aplicaciones de un esquema - que se menciona como de procedencia platónica-, el cual es, más allá de ciertas diversidades, básicamente el mismo que se tiene en cuenta en Cat. 12 (2). En efecto, este último esquema (Cat. 12 (2)) resulta demasiado rudimentario para lo que debería justificar. En mi opinión, Aristóteles no logra especificar un concepto de prioridad adecuado a su uso de la prioridad en la noción de prôte ousía; y me parece dudoso que haya considerado Cat. 12 (2) como ese concepto. La aplicación al marco de las categorías y al de potencia-acto, en cambio, hace que el esquema lógicosemántico de Platón, aludido en Metaph. V 11 (4), adquiera una muy precisa determinación ontológica. Vigo busca establecer la diferencia entre ambas prioridades atendiendo a que $\mathrm{Me}$ taph. V 11 (4) constituiría un "criterio de índole básicamente ontológica que parece vincularse más —a diferencia de Cat. 12 (2), donde este autor observa la enunciación de un vínculo 'lógico-semántico' entre dos proposiciones existenciales- con

${ }^{22}$ Cfr. Vigo [1989], p. 95. 
relaciones in rebus del tipo de la relación entre el fundamento y lo fundado, según los casos". ${ }^{23}$ Este autor sostiene que la diferencia entre Cat. 12 (2) y Metaph. V 11 (4) residiría en que Cat. 12 (2) tendría la tarea de formular un "criterio de reconocimiento" de la misma relación que en Metaph. V 11 (4) se formula expresamente. Vigo cree ver confirmada esta distinción en el hecho de que Cat. 12 (5) destacaría la conexión causal como condición necesaria para establecer, en el caso de una relación de biimplicación, una anterioridad por naturaleza (phýsei). La comparación entre Cat. 12 (2) y (5) muestra, efectivamente, que para establecer la asimetría entre dos hechos $(X \mathrm{e}$ $Y)$ en cuanto a la relación de antero-posterioridad es necesario determinar si $X$ e $Y$ son irreversibles (el caso de (2)); pero en casos como el de (5), donde $X$ e $Y$ son reversibles semántica y existencialmente, es factible aplicar otro criterio para fijar la anterioridad: el causal. Así, por ejemplo, el concepto del dos implica la anterioridad existencial del uno, pero no viceversa (no reversibilidad). Cuando dos eventos son simultáneos y existencialmente reversibles, como, por ejemplo, la existencia de un hombre y el enunciado verdadero que afirma la existencia de ese hombre, podemos establecer la anterioridad de uno con relación al otro considerando la relación causal que los vincula. En este caso, la existencia de $x$ es tomada por Aristóteles como el factor explicativo que es condición sine qua non de la verdad del enunciado que afirma la existencia de $x$ (cfr. Cat. 12, 14b 10-12).

Por consiguiente, de esa consideración comparativa surge que la falta de asimetría, esto es, la simetría entre dos hechos, no es un dato suficiente, sino sólo necesario para determinar dos entidades en cuanto a la relación de anterioridad. Un corolario de esto reside en que la simetría no constituye un criterio suficiente para determinar la simultaneidad (como lo muestra (5)). ${ }^{24}$ Vigo critica, con razón, la equiparación entre Cat. 12 (5) y Metaph. V 11 (1), sugerida por Ross. ${ }^{25}$ Pero una cuestión distinta es

${ }^{23}$ Cfr. Vigo [1989], p. 95.

${ }^{24}$ Cfr. Vigo [1989], p. 95 fin.

${ }^{25}$ Cfr. Vigo [1989], p. 95 n. 13; Ross [1958], I, p. 317. 
si, efectivamente, Cat. 12 (5) representa una vinculación entre Cat. 12 (2) y Metaph. V 11 (4), como afirma Vigo. ${ }^{26}$ Me parece, por el contrario, que las aplicaciones aristotélicas de Metaph. V 11 (4) no pueden ser explicadas a partir de Cat. $12(2)+(5)$. En efecto, la prioridad de la sustancia, en el esquema de las categorías y en el de potencia-acto, dentro del segundo modelo ontológico de Aristóteles, no se explica correctamente como la anterioridad de algo que es causa de otra cosa. La sustancia no es anterior en cuanto causa de las determinaciones accidentales (posteriores qua causadas), sino que es anterior en cuanto completamente determinada en relación con propiedades que pueden atribuírsele, pero nunca contribuir a la determinación de su identidad ya que no enuncian su qué, sino sólo su cuánto, su cuál, su relación con algo etc. Si además Metaph. V 11 (4) constituyera un "criterio de reconocimiento" (Vigo) de la relación expresada en Cat. 12 (2), entonces los dos esquemas de V 11 (4) se reducirían a modalidades para afirmar la implicación de existencia. Sin embargo, en ambos se afirma que hay dos tipos de entidades cuyas determinaciones no pueden 'deducirse' directamente del concepto de otras.

\section{Prioridad lógica y ontológica de la sustancia primera}

Hasta aquí creo haber puesto un poco más en claro una herramienta principal que incide en el origen y en la fundamentación del esencialismo aristotélico. La noción más básica de éste debe localizarse, efectivamente, en la distinción entre esencia y accidente que se formula recurriendo al concepto de prioridad junto a otras herramientas de análisis asociadas. En mi diagnóstico he tratado de argumentar a favor de que el concepto de prioridad que sostiene la posición de la sustancia madura en Metaph. VII no es explicable recurriendo a un cambio de posición de una prioridad natural a otra lógica, sino que es simplemente el resultado del esclarecimiento de la prioridad de la ousía en el esquema de las categorías ya operante en el primer modelo ontológico de Aristóteles, aunque con las limitaciones señaladas.

${ }^{26}$ Cfr. Vigo [1989], p. 95 n. 13. 
De los parágrafos anteriores puede concluirse que la doctrina de la sustancia representa la alternativa teórica - formulada a base de la doctrina de las categorías y del concepto operativo de prioridad allí introducido - a la doctrina platónica de las ideas y los principios más allá de que Aristóteles conserve las prerrogativas básicas de las ideas platónicas en sus propias entidades por sí. Al descubrimiento de éstas sirve la clasificación de predicados. Pero al reformular - mediante un esclarecimiento obtenido a partir de datos que ofrecen la función predicativa y las distintas clases de predicados que entran en ella- la prioridad que organiza la relación entre las entidades, se desemboca en un análisis categorial de la sustancia y en la explicación de su estructura por medio de partes completamente distintas de los elementos platónicos.

Después de haber intentado clarificar cuál es el concepto de prioridad que permite articular la primera doctrina de las categorías, lo que me resta por hacer en este parágrafo es tratar de extraer algunas consecuencias para el concepto mismo de sustancia primera. Lo que he sostenido hasta aquí es que la posición de la sustancia primera en la primera metafísica de Aristóteles sólo alcanza a recibir una fundamentación adecuada al concepto mismo de la prioridad de la sustancia individual mediante una noción de prioridad como la que expone Metaph. V 11 (4iia). He propuesto denominar a ésta 'prioridad ontológica' distinguiéndola tanto de (i) una "prioridad meramente existencial' - como la clasificada en Cat. 12 (2) - , cuanto de (ii) una 'prioridad meramente conceptual' — como la de Metaph. V 11 (2Aii) - que le corresponde a la noción de los atributos.

Anteriormente he sostenido que Cat. 12 (2) y Metaph. V 11 (4) no son equiparables; reafirmar esto es importante para despejar la inquietud que podría plantearse advirtiendo que la prioridad de la sustancia - siempre en el marco de Categorías - está suficientemente explicada mediante Cat. 12 (2). Pero, junto a ello, la cosa se complicaría aún más para el diagnóstico emitido sobre los límites de fundamentación y algunos problemas del concepto de sustancia y la relación objeto-atributo dentro del primer modelo ontológico de Aristóteles si fuera efectivamente 
correcto que el concepto de prioridad de Cat. 12 (2) y Metaph. $\mathrm{V} 11$ (4) son idénticos. Ahora bien, ni una ni otra cosa son interpretaciones correctas de la posición aristotélica.

Difícilmente puede Aristóteles explicar la prioridad operante en su concepto de sustancia primera apelando al aspecto meramente existencial - en una aplicación literal de lo que Owen llamó 'prioridad natural'-, ya que no es tan sólo la existencia del objeto lo que estrictamente presuponen los accidentes en la teoría de los compuestos accidentales. Esta aplicación desplazaría la argumentación de Categorías en lo que constituye su teoría de la prioridad de la sustancia individual formulada mediante la teoría de la predicación expuesta en Cat. 2. Según esto, las sustancias individuales son el tipo de entidades que se requiere para dar un sustento a la predicación accidental estándar. Las sustancias individuales no resultan así meramente anteriores en cuanto su existencia es necesaria y se halla implicada directamente en una proposición existencial. Los enunciados estándar, cuya condición de posibilidad Categorías se propone explicar, no son aprehendidos por Aristóteles como enunciados existenciales, sino que son explicados como distintos tipos de cualificación del objeto. La prioridad de la sustancia individual no puede fundamentarse entonces por recurso al esquema de la no reversibilidad existencial, ni puede denominarse por lo que Owen entiende como prioridad natural. Sin embargo, en Categorías, Aristóteles no dispone de otra noción, y eso marca nuevamente las limitaciones reflexivas del primer modelo metafísico del estagirita.

En segundo lugar - recalcando algo de lo que antes dije sobre la diferencia entre Cat. 12 (2) y Metaph. V 11 (4)-, un dato que no puede pasarse por alto está dado por el hecho de que Cat. 12 (2) afirma únicamente que puede deducirse la existencia de algo anterior a otra cosa en cuanto se halla implicado en su propio concepto. Esta noción no es equiparable ni puede utilizarse como un criterio válido constantemente para reconocer una entidad cuya determinación es anterior a otra. Lo que no puede ser sin lo anterior, dentro de esta última noción, no es algo que remita sólo existencialmente a lo an- 
terior, sino, más bien, algo cuya definición presupone la de lo anterior. Aristóteles aplica esta noción a dos esquemas distintos: Metaph. V 11 (4iia, iib). Así, la materia resulta potencialmente anterior a la forma en cuanto no es meramente anterior desde el punto de vista temporal, sino en cuanto su propio concepto puede ser analizado como anterior al de la forma en la coordenada de la potencia; por ejemplo, la naturaleza de los materiales requeridos para el desempeño de la función de una mano es potencialmente anterior a la forma que determina la función de ese órgano compuesto. Pero esos materiales son posteriores a la forma materializada (énhylon) en la coordenada del acto, ya que como tales no existen separados ni tampoco pueden hacerlo conservando estrictamente su identidad y su función una vez que son separados (arrancados) del todo al que pertenecen (cfr. 'mutilado' Metaph. V 27). Ahora bien, esa dependencia funcional e inseparabilidad existencial no implica que no pueda considerarse que una parte material de un sýnolon no pueda ser de ninguna manera sin la forma; pero sólo puede ser potencialmente separada, y ello restringe la anterioridad de la parte física de un sýnolon a lo potencialmente anterior en el concepto. Dicho de otra manera, no hay simetría en esta relación ya que no podemos obtener la forma actual a partir de los materiales, sino sólo los materiales a partir de la forma. ${ }^{27}$

Pero no me ocupa aquí tanto este caso - aunque si no valiera para (iib) lo dicho para (iia) debería suponerse, de manera poco plausible, un doble significado en las aplicaciones del mismo concepto o correría peligro la interpretación propuesta-, cuanto más bien el de (iia). Decididamente no tenemos allí el concepto de lo que Owen llamó 'prioridad natural'. La aplicación del esquema académico general de anterior-posterior al análisis de compuestos accidentales puede considerarse que cristaliza en el uso ya reclamado de Metaph. V 11 (4iia); pero también colabora en la explicación del concepto de prioridad de la sustancia

${ }^{27}$ Para la aplicación de este concepto de anterioridad a los compuestos hilemórficos cfr. Makin [2003], p. 214 y ss., 218 y s. n. 15, 225 y ss. La relación entre forma y partes materiales presenta aristas muy delicadas que no me propongo considerar en este lugar y que es intensamente discutida en la actualidad; cfr. entre otros, Gill [1991], en especial 4 (pero en general 4-7). 
primera el significado distinguido en Metaph. V 11 (2Aii), que denominé 'prioridad lógica'. Anteriormente consideré ya este concepto destacando la contraposición en que él se halla con la 'prioridad existencial'. Metaph. V 11 (4iia) y V 11 (2Aii) explican que la prioridad de la sustancia primera es la de algo que debe ser determinado como condición para que otras propiedades puedan tener una determinación (no ser indefinidas). Esa determinación está presupuesta en la definición de aquellas propiedades como el objeto en un atributo. Esta aplicación de los dos conceptos, ciertamente, no establece en las Categorías un esquema como el de la unidad referencial; pero su ausencia parece tener que explicarse, antes bien que por un cambio de posición (Owen), más concretamente en virtud de la limitación de un desarrollo explicativo e igualmente debido a la limitación impuesta al esclarecimiento de la relación objeto-atributo por el dominio que sobre esa relación ejerce la clase de predicación seren. Ésta condiciona la prioridad efectiva de la sustancia primera llegando casi a equiparar su propio estatus al de los atributos como consecuencia tanto de una insuficiente explicación de la posterioridad de los accidentes - ya que esa explicación se formula allí apelando meramente al dato fáctico de la dependencia existencial de los atributos- ${ }^{28}$ cuanto también por el hecho de que la determinación de la sustancia se hace por una vía común a ella y a los atributos, es decir, la definición, apelando a un modelo esencialista de raigambre platónica que mediante el 'qué es' determina entidades independientes sin función de objeto, o sea, entidades que no desempeñan la función de substrato de propiedades, que es propia de la noción aristotélica de 'sustancia'. ${ }^{29}$ Con otras palabras, el primer modelo ontológico

${ }^{28}$ Esto es señalado por Tugendhat $\left[1988^{4}\right], \S 6$ y s., como una diferencia central entre Cat. y Metaph. VII 1 en lo que hace a la relación entre la ousía y las restantes categorías.

${ }^{29}$ En "La paradoja de la esencia" he tratado de caracterizar este problema como originado en el fundamento esencialista de la prioridad de la ousía. Allí me ocupo igualmente de poner en esta perspectiva las dos diferentes clases de dependencia (de universales sustanciales respecto de sustancias individuales, por un lado, y de entidades no sustanciales respecto de sustancias individuales, por otro lado) que menciono a continuación en el texto. 
de Aristóteles carece de una doctrina de la definición adecuada a la característica distintiva de los objetos sustanciales. Ese primer modelo reduplica el orden de sustancias (sustancias primeras y segundas) para tratar de salvar esta dificultad en la definición de los individuos. Pero la reduplicación se hace sin justificar el estatus sustancial de entidades postuladas para explicar la identidad de otras ya completamente determinadas y no legitima teóricamente la determinación que se admite para los individuos fundamentales de esta metafísica.

Esta limitación no se supera con un cambio en la noción de prioridad, pues la prioridad del individuo sustancial de Categorías es únicamente posible gracias a la operación de los dos significados de prioridad que, con la radicalización del planteamiento, dan lugar en Metaph. VII a la inauguración de la cuestión de la ousía y a la explicación referencial de la unidad de los múltiples significados de 'ser'. Esta explicación implica una inmediata 'desencialización' en la determinación de la identidad de las distintas clases de entidades, tanto en la determinación de la sustancia, que no puede definirse meramente como un 'qué es' platónico, cuanto en la determinación de los accidentes, que en cuanto atributos no son estrictamente definibles mediante una caracterización acuñada para ser aplicada originalmente a entidades por sí. La innovación mayor que trae el concepto de sustancia en la metafísica madura no se halla allí donde Owen creyó localizarlo, sino que, más bien, proviene de una fundamentación de la relación de dependencia que guardan los universales de la primera categoría con respecto a los individuos. Ese desarrollo da lugar a la operación de un nuevo concepto de prioridad aplicado a la vinculación entre la materia y la forma que representan las nuevas partes del objeto en el segundo modelo ontológico del estagirita. ${ }^{30}$

Si esto es correcto, la solución aristotélica a la aprehensión de la definición de las entidades bajo un modelo platónico ${ }^{31}$ no

\footnotetext{
${ }^{30}$ Para una sugerencia en esta dirección acerca del cambio de posición de la metafísica primera a la madura de Aristóteles, cfr. Rapp [1995], pp. 95 y ss.

${ }^{31}$ Esto demarca la base de lo que llamo 'paradoja de la esencia'.
} 
tiene lugar primeramente a través de la formulación semántica relativa a la unidad referencial de 'ser', pues esta formulación representaría, antes bien que un cambio con respecto a la posición original del ser categorial, un esclarecimiento teórico formulado en continuidad con la posición original de las categorías. La 'paradoja de la esencia' consiste básicamente, en cambio, en una aprehensión esencialista del objeto, que no da lugar a la noción madura de la sustancia aristotélica entendida como un sujeto (hypokeímenon) determinado (tóde ti). La solución de esa paradoja y la superación de su esencialismo intrínseco coinciden con la formulación teóricamente fundamentada de la prioridad de la forma sustancial cuyo concepto representa el esclarecimiento aristotélico de la prioridad del individuo de las Categorías. Recién entonces la prioridad del individuo alcanza la fundamentación que en Categorías le faltaba en el orden de la identidad y la unidad. Para operar esta teoría hace falta un concepto de prioridad que se tiene básicamente en Metaph. V 11 (4iib). En el último de los casos allí considerados se incluye la anterioridad sólo potencial de la materia; esta anterioridad implica que la forma es actualmente primaria en cuanto sirve para explicar la estructura del compuesto. Pero esto involucra, de hecho, una inversión del orden de prioridades establecido en Categorías a partir de la sola consideración de la relación de dependencia de los atributos con respecto a los objetos. Ese orden se modifica cuando pasa a considerarse el fundamento de la identidad y la unidad de la sustancia individual, para lo cual es preciso apelar a una relación de dependencia diferente. En Categorías, en cambio, la fundamentación de la prioridad de los individuos ante los universales sustanciales no va más allá de la caracterización de los primeros mediante el rasgo del 'no decirse de nada diferente como de su sujeto', lo que representa una descripción tomada de la distinción del objeto frente a propiedades atributivas (accidentes). Pero con ello no se ha elaborado una noción sustancial válida para que los universales sustanciales dependan de individuos, pues en su primer modelo ontológico Aristóteles no reconoce la contribución a la identidad y a la unidad del individuo que ejercen propiedades analizadas bajo la 
categoría de sortales en función identificadora. La "paradoja de la esencia' da lugar a que la prioridad de las sustancias primeras resulte justificada sólo mediante una errónea equiparación de las dos distintas relaciones fundamentales de dependencia. Este movimiento teórico es solidario con una aprehensión esencialista del objeto, que coincide con la suposición teóricamente injustificada de la individualización del objeto tomado como algo ya específicamente determinado.

Sin entrar ahora en una reconstrucción conceptual detallada de las Categorías, es posible fortalecer las observaciones anteriores atendiendo a que el denominado "pasaje de la primacía' (Cat. 5, 2a 34-b 6) destaca a las sustancias individuales como condición para que existan tanto cosas que se dicen-de, como otras que son-en. El 'pasaje del sujeto' (2b 29-3a 6) especifica a géneros y especies como los portadores de la información adecuada para conocer lo que es una sustancia. Esa función informativa hace de géneros y especies 'sustancias', que por ello mismo se diversifican frente a propiedades no sustanciales. Pero Aristóteles no analiza la relación que supone esa especificación. En el último pasaje citado, su propósito es explicar que mediante la generalización puede decirse de los universales todo aquello que se predica del individuo sustancial. Que el universal aquí considerado tiene el estatus de una sustancia es únicamente justificado a través de su carácter informativo. Pero la paradoja reside en que esa información específica que da el universal sustancial forma parte de la noción del individuo determinado de manera esencialista a partir del qué-es, con lo cual la función del universal del mismo tipo (sustancial) queda sin justificar y su postulación resulta teóricamente superflua.

\section{BIBLIOGRAFÍA}

\section{Al. Aristóteles}

, 1964, Analytica priora et posteriora, W.D. Ross ed., Oxford University Press, Oxford.

Oxford. 
1949, Categoriae et Liber de Interpretatione, L. MinioPaluello ed., Oxford University Press, Oxford.

1972 (1965), De generatione animalium, H.J. Drossart Lulofs ed., Oxford University Press, Oxford.

, 1975, Il "De ideis" di Aristotele e la teoria platonica delle idee, Dieter Harlfinger (ed. del texto), Walter Leszl (autor del estudio crítico que acompaña al volumen), Leo S. Olschki, Florencia.

Aristóteles, 1993 (1957), Les Parties des Animaux, Pierre Louis ed., Les Belles Lettres, París.

1957, Metaphysica, W. Jaeger ed., Oxford University Press, Oxford.

— , 1950, Physica, W.D. Ross ed., Oxford University Press, Oxford.

—— 1991 (1970), Topica et Sophistici elenchi, W.D. Ross ed., Oxford University Press, Oxford.

\section{A2. Platón}

1900-1907 (varias reediciones), Opera, 5 vol., I. Burnet ed., Oxford University Press, Oxford.

Bl. Comentarios antiguos (Comentaria in Aristotelem Graeca $(\mathrm{CAG}))$.

\section{Categorías}

Amonio, 1895, in Aristotelis Categorias commentarius, A. Busse ed., Georg Reimer, Berlín.

Simplicio, 1907, in Aristotelis Categorias commentarium, C. Kalbfleisch ed., Georg Reimer, Berlín.

B2. Comentarios modernos

\section{Analíticos Segundos}

Barnes, Jonathan, $1993^{2}$, Aristotle Posterior Analytics, trad. y comentario de J. Barnes, Oxford University Press, Oxford.

Detel, Wolfgang, 1993, Aristoteles, Analytica Posteriora, trad. y comentario de W. Detel, 2 vols., Aristoteles Werke en traducción al alemán, ed. H. Flashar, vol. 3, parte II, Akademie Verlag, Berlín. 
Ross, W.D., 1949, Aristotle's Prior and Posterior Analytics, texto revisado con introducción y comentario de W.D. Ross, Oxford University Press, Oxford.

Categorías

Ackrill, John L., 1963, Aristotle's Categories and De Interpretatione, trad. y notas de J.L. Ackrill, Oxford University Press, Oxford.

Oehler, Klaus, 1997 (1983), Aristoteles, Kategorien, trad. y comentario de K. Oehler, Aristoteles Werke en traducción al alemán 1/I, Akademie Verlag, Berlín.

\section{Metafísica}

Annas, Julia, 1988 (1976), Aristotle's Metaphysics. Books M and N, trad., introducción y notas de J. Annas, Oxford University Press, Nueva York.

Reale, Giovani, 1993, Aristotele, Metafisica, 3 vols., Vita e Pensiero, Milán.

Ross, W.D., 1958, Aristotle's Metaphysics, texto revisado con introducción y comentario de W.D. Ross., 2 vols., Oxford University Press, Oxford.

C. ÍNDICE

Bonitz, Hermann, $1955^{2}$ (1870), Index Aristotelicus, Akademische u. Verlagsanstalt, Graz.

\section{LiTERATURA ESPECÍfICA}

Berti, Enrico, 1975, "Priorità logica e ontologica fra i generi di sostanza in Aristotele", en E. Berti 1975, Studi aristotelici, L.U. Japadre, El Aguila (Italia), pp. 261-273.

Bolton, Robert, 1976, "Essentialism and Semantic Theory in Aristotle", Philosophical Review, no. 85, pp. 14-544.

Calvo, Tomás, 1991, "La fórmula kath'autó y las categorías: a vueltas con Metafisica V 7", Methexis, IV, pp. 39-57.

Cattanei, Elisabetta, 1996, Enti matematici e metafisica. Platone l'Accademia e Aristotele a confronto, Vita e Pensiero, Milán.

__ 1990, "I metodi della metafisica platonico-accademica 'generalizzante' ed 'elementarizante' nei libri 'M' e 'N' della Metafisica di Aristotele", Rivista di Filososofia neo-scolastica, LXXXII 2-3, pp. 183-213. 
Cleary, John J., 1995, Aristotle and Mathematics. Aporetic Method in Cosmology and Metaphysics, Philosophia Antiqua LXVII, Brill, Leiden/Nueva York/Colonia.

Code, Alan, 1986, "Aristotle on Essence and Accident", en R.E. Grandy y R. Warner (comps.), Philosophical Grounds of Rationality, Oxford/Nueva York, pp. 411-444.

— 1982, "On the Origins of some Aristotelian Theses about Predication", en J. Bogen y J.E. McGuire (comps.), How Things Are, Dordrecht/Boston, pp. 101-131.

Cohen, S.M., "Plato's Method of Division", en J.M.E. Moravesik (comp.), Patterns in Plato's Thought, Reidel, Dordrecht/Boston, pp. 181-191.

Dancy, R.M., 1975, “On Some of Aristotle's First Thought about Substances", Philosophical Review, 84, pp. 338-373.

Detel, Wolfgang, 1998a, "Eine terminologische Rekonstruktion von Arist. Cat. 1-5", en R. Enskat (comp.), Amicus Plato magis amica veritas. Feschrift für Wolfgang Wieland, De Gruyter, Berlín/Nueva York, 1998, pp. 60-81.

—_, 1998b, "Metaphysik und Wissenschaftslehre bei Aristoteles", Internantionale Zeitschrift für Philosophie, 2, pp. 199-229.

Ferejohn, Michael T., 1981, "Aristotle on Necessary Truth and Logical Priority", Philosophical Quarterly, 18/4, pp. 285-293.

Fine, Gail, 1998 (1993), On Ideas. Aristotle's Criticism of Plato's Theory of Forms, Oxford University Press, Nueva York.

Frede, Michael, 1987a, "Categories in Aristotle", en M. Frede, Essays in Ancient Philosophy, Oxford University Press, Minnéapolis, pp. 29-48.

Gaiser, Konrad, 19682 ${ }^{2}$ Platons ungeschriebene Lehre. Studien zur systematischen und geschichtlichen Begründung der Wissenschaft in der Platonischen Schule (Anhang: Testimonia Platonica), Klett, Stuttgart.

Gill, Mary-Louise, 1991 (1989), Aristotle on Substance. The Paradox of Unity, Princeton University Press, Princeton (Nueva Jersey).

Happ, Heinz, 1971, Hyle. Studien zum aristotelischen MaterieBegriff, De Gruyter, Berlín/Nueva York.

Kapp, Ernst, 1920, "Die Kategorienlehre in der aristotelischen Topik", en E. Kapp, Ausgewählte Schriften, 1968, ed. H. y I. Diller, Berlín, pp. 215-253.

Lee, E.N., 1972, "Plato on Negation and Not-Being in the Sophist", Philosophical Review, LXXXI, 3, pp. 267-303. 
Lewis, Frank, 1991, Substance and Predication in Aristotle, Cambridge University Press, Nueva York.

Lowe, E.J., 1989, Kinds of Being. A Study of Individuation, Identity and the Logic of Sortal Terms, Oxford University Press, Oxford.

Makin, Stephen, 2003, "What does Aristotle mean by Priority in Substance", Oxford Studies in Ancient Philosophy, XXIX, pp. 209238.

Marcos de Pinoti, Graciela, 1995, "Autour de la distinction entre Eidos et Meros dans le Politique de Platon (262a 5-263e 1)", en C. Rowe (comp.), Reading the Stateman, Academia Verlag, San Agustín (Alemania), pp. 155-161.

Mié, Fabián, 2001, "Identidad, definición y clases naturales en el método platónico de la división", Methexis, XIV, pp. 87-99.

Mié, Fabián, 2002, Recensión de M.V. Wedin, Aristotle's Theory of Substance. The Categories and Metaphysics Zeta, Oxford University Press, Nueva York, Ordia prima I, pp. 161-165.

Moravcsik, J.M.E., 1973, "Plato's Method of Division", en J.M.E. Moravcsik (comp.) Patterns in Plato's Thought, Reidel, Dordrecht/Boston, pp. 158-180.

O'Brien, Denis, 1995, Le non-être. Deux Études sur le Sophiste de Platon, Academia Verlag, San Agustín (Alemania).

Owen, G.E.L., 1960, "Logic and Metaphysics in Some Earlier Works of Aristotle", en I. Düring y G.E.L. Owen (comps.), Aristotle and Plato in the Mid-Fourth Century, Gotenburgo, pp. 163-190.

— , 1971, "Plato on Not-Being", en G. Vlastos (comp.), Plato I. Metaphysics and Epistemology, A Collection of Critical Essays, Garden City, pp. 223-267.

Rapp, Christof, 1995, "Allgemeines konkret —Ein Beitrag zum Verständnis der Aristotelischen Substanzlehre", Philosophisches Jahrbuch, 102, pp. 83-100.

——, 1995a, Identität, Persistenz und Substanzialität. Untersuchungen zum Verhältnis von sortalen Termen und Aristotelischer Substanz, Alber, Friburgo de Brisgovia/Munich.

Sorabji, Richard, 1980, Necessity, Cause and Blame. Perspectives on Aristotle's Theory, Duckworth, Londres.

Szaif, Jan, 1998², Platons Begriff der Wahrheit, Alber, Friburgo de Brisgovia/Munich.

Tugendhat, Ernst, $1988^{4}$, TI KATA TINOS. Eine Untersuchung zu Ursprung und Struktur aristotelischer Grundbegriffe, Alber, Friburgo de Brisgovia/Munich. 
Vigo, Alejandro Gustavo, 1990, "Prioridad ontológica y prioridad lógica en la doctrina aristotélica de la sustancia", Philosophica, 13, pp. 175-199.

—_ 1989, "Prioridad y prioridad ontológica según Aristóteles", Philosophica, 12, pp. 89-113.

Wedin, Michael V., 2000, Aristotle's Theory of Substance. The Categories and Metaphysics Zeta, Oxford University Press, Nueva York.

White, Nicholas P., 1972, "Origins of Aristotle's Essentialism", Review of Metaphysics, XXVI/1, pp. 57-85.

Wilpert, Paul, 1949, Zwei aristotelische Frühschriften über die Ideenlehre, Druck und Verlag von Josef Habbel, Regensburgo.

Witt, Charlotte, 2000 (1994), "The Priority of Actuality in Aristotle", en T. Scaltsas, D. Charles, y M.L. Gill (comps.), Unity, Identity, and Explanation in Aristotle's Metaphysics, Oxford University Press, Nueva York, pp. 215-228.

Recibido el 10 de enero de 2003; revisado el 23 de junio de 2003; aprobado el 2 de julio de 2003. 